Bryn Mawr College

Scholarship, Research, and Creative Work at Bryn Mawr

College

Physics Faculty Research and Scholarship

Physics

2012

\title{
Sheared-flow induced confinement transition in a linear magnetized plasma
}

S. Zhou

W.W. Heidbrink

H. Boehmer

R.McWilliams

T. A. Carter

See next page for additional authors

Let us know how access to this document benefits you.

Follow this and additional works at: http://repository.brynmawr.edu/physics_pubs

Part of the Physics Commons

\section{Custom Citation}

S. Zhou, W. W. Heidbrink, H. Boehmer, R. McWilliams, T. A. Carter, S. Vincena, B. Friedman, D. Schaffner. 2012. Sheared-flow induced confinement transition in a linear magnetized plasma. Phys. Plasmas 19, 012116.

This paper is posted at Scholarship, Research, and Creative Work at Bryn Mawr College. http://repository.brynmawr.edu/physics_pubs/83

For more information, please contact repository@brynmawr.edu. 
Authors

S. Zhou, W. W. Heidbrink, H. Boehmer, R. McWilliams, T. A. Carter, S. Vincena, B. Friedman, and David A. Schaffner 


\title{
Sheared-flow induced confinement transition in a linear magnetized plasma
}

\author{
S. Zhou, ${ }^{1}$ W. W. Heidbrink, ${ }^{1}$ H. Boehmer,${ }^{1}$ R. McWilliams, ${ }^{1}$ T. A. Carter, ${ }^{2}$ S. Vincena,${ }^{2}$ \\ B. Friedman, ${ }^{2}$ and D. Schaffner ${ }^{2}$ \\ ${ }^{1}$ Department of Physics and Astronomy, University of California, Irvine, California 92697, USA \\ ${ }^{2}$ Department of Physics and Astronomy, University of California, Los Angeles, California 90095, USA
}

(Received 22 September 2011; accepted 12 December 2011; published online 23 January 2012)

A magnetized plasma cylinder (12 $\mathrm{cm}$ in diameter) is induced by an annular shape obstacle at the Large Plasma Device [W. Gekelman, H. Pfister, Z. Lucky, J. Bamber, D. Leneman, and J. Maggs, Rev. Sci. Instrum. 62, 2875 (1991)]. Sheared azimuthal flow is driven at the edge of the plasma cylinder through edge biasing. Strong fluctuations of density and potential $\left(\delta n / n \sim e \delta \phi / k T_{e} \sim 0.5\right)$ are observed at the plasma edge, accompanied by a large density gradient $\left(L_{n}=|\nabla \ln n|^{-1} \sim 2 \mathrm{~cm}\right)$ and shearing rate $(\gamma \sim 300 \mathrm{kHz})$. Edge turbulence and cross-field transport are modified by changing the bias voltage $\left(V_{\text {bias }}\right)$ on the obstacle and the axial magnetic field $\left(B_{z}\right)$ strength. In cases with low $V_{\text {bias }}$ and large $B_{z}$, improved plasma confinement is observed, along with steeper edge density gradients. The radially sheared flow induced by $E \times B$ drift dramatically changes the cross-phase between density and potential fluctuations, which causes the wave-induced particle flux to reverse its direction across the shear layer. In cases with higher bias voltage or smaller $B_{z}$, large radial transport and rapid depletion of the central plasma density are observed. Two-dimensional cross-correlation measurement shows that a mode with azimuthal mode number $m=1$ and large radial correlation length dominates the outward transport in these cases. Linear analysis based on a two-fluid Braginskii model suggests that the fluctuations are driven by both density gradient (drift wave like) and flow shear (Kelvin-Helmholtz like) at the plasma edge. (C) 2012 American Institute of Physics. [doi:10.1063/1.3677361]

\section{INTRODUCTION}

Modification of the plasma edge turbulence and turbulent transport has attracted continuous interest since the discovery of the high-confinement mode (H-mode), where a strong and sudden change in plasma characteristics, leading to improved energy confinement, is observed. H-mode in tokamaks $^{1}$ is triggered mostly when the heating power exceeds a threshold. Many experiments ${ }^{2}$ and theories ${ }^{3}$ ascribed the improved confinement to the formation of the steady sheared flows, which can stabilize plasma turbulence and form a transport barrier. The sheared flow in plasmas can be spontaneously generated by nonlinear interaction of the edge turbulence (zonal flows), or induced by externally applied radial electric fields $(E \times B$ drift). It was first demonstrated in the Continuous Current Tokamak (CCT) experiments ${ }^{4}$ that a sharp transport barrier, accompanied by an $\mathrm{H}$-mode like state, is triggered by applied radial electric fields. Subsequently, many experiments have used external bias to study modification of the plasma turbulence and transport. $^{5-8}$

Recent experiments ${ }^{8-10}$ in the upgraded Large Plasma Device $^{11}$ (LAPD) at the University of California, Los Angeles (UCLA) have studied the effect of the sheared flow on the edge turbulence and turbulent transport by biasing a section of the vacuum chamber wall. Linear and nonlinear simulations to model the plasma instabilities based on LAPD parameters are also developed. ${ }^{12,13}$ In the current experiment, an annular-shaped obstacle with inner radius of $6 \mathrm{~cm}$ is placed concentrically with the main plasma of LAPD. The obstacle partially blocks the plasma source and induces a plasma column $12 \mathrm{~cm}$ in diameter downstream. The obstacle is biased by an external power supply, leading to sheared $E \times B$ drift at the edge of the plasma column. Modifications of the plasma characteristics and turbulent transport by changing the amplitude of the bias on the obstacle $\left(V_{\text {bias }}\right)$, the axial magnetic field $\left(B_{z}\right)$ strength and the plasma species (helium or neon) are fully documented and studied in this experiment. A companion paper ${ }^{14}$ discusses the effect of these fluctuations on the transport of energetic ions.

Here we summarize the primary results reported in this paper. Two very different states of plasma characteristics are observed with varied control parameters $\left(V_{\text {bias }}, B_{z}\right.$ and plasma species). Compared to the plasma characteristics with the obstacle floating, in one parameter regime, improved plasma confinement is observed during the $3 \mathrm{~ms}$ pulsed biasing time, featuring a steeper edge density gradient and increased plasma density. In another parameter regime, the plasma density drops significantly during the biasing time and large radially outward transport is observed. Such cases are called plasma depletion for the rest of this paper. It is evident that the velocity and shearing rate of the radially sheared azimuthal flow plays an important role in both states. The plasma depletion is also found to be associated with relatively large ion sound radius $\left(\rho_{s}\right)$. The plasma density evolution during the biasing time is explained clearly by the particle flux profiles and the density and potential fluctuation spectra. For cases with improved confinement, significant change in the particle flux direction occurs at the sheared-flow layer, leading to reversed particle flux profiles. The two-dimensional correlation functions of density and potential fluctuations show that the cross 
phase between density and potential is changed by $\sim 180^{\circ}$ across the sheared layer, which explains the reversed direction of the particle flux in these cases. For cases with plasma depletion, large radially outward particle flux is observed, accompanied by low mumber $(m \sim 1)$ modes with large radial correlation scale length (comparable to the scale size of the plasma column). Linear analysis based on the Braginskii two-fluid model suggests that the edge turbulence is driven by both density gradient (drift wave like instability) and flow shear (Kelvin-Helmholtz like instability).

The organization of this paper is as follows: The experimental setup and basic plasma parameters are introduced in Sec. II. Major experimental results including the particle flux profiles and the spectral analysis of the edge turbulence are reported in Sec. III. In Sec. IV, the possible wave modes are discussed and linear analyses of the wave dispersion relations and growth rates based on a two-fluid model are presented. Conclusions are drawn in Sec. V.

\section{EXPERIMENTAL SETUP}

The experiments were performed in plasmas generated in the upgraded LAPD at the University of California, Los Angeles. The LAPD is an 20 -m-long, 1 -m-diameter cylindrical vacuum chamber, coaxial with 56 solenoid magnetic field coils. Pulsed plasmas with $\sim 10 \mathrm{~ms}$ duration are created using a barium oxide coated cathode source at $1 \mathrm{~Hz}$ repetition rate. During the pulse time, fast electrons with energy of $\sim 50 \mathrm{eV}$ are created. These electrons flow along the axial magnetic field line through the gridded anode and ionize the working gas in the main chamber to form a plasma column. The plasma column has a diameter of $\leq 70 \mathrm{~cm}$. The axial magnetic field in this experiment is uniform and ranges from 600 $\mathrm{G}$ to $1800 \mathrm{G}$. Typical plasma parameters during the active discharge are plasma density $n_{e} \sim 2.5 \times 10^{12} \mathrm{~cm}^{-3}$, electron temperature $T_{e} \sim 5 \mathrm{eV}$, ion temperature $T_{i} \sim 1 \mathrm{eV}$. The working gases used in this experiment are helium and neon. The experimental setup is shown schematically in Fig. 1. A copper, annular obstacle is inserted concentrically in the main plasma. The obstacle has inner diameter of $12 \mathrm{~cm}$ and outer diameter of $20 \mathrm{~cm}$. Downstream from the obstacle, a plasma column with diameter of $12 \mathrm{~cm}$ and steep edge density gradient is formed. The obstacle shaft and the LAPD anode are connected by a bias circuit including acapacitor bank and an IGBT (Insulated Gate Bipolar Transistor) switch. A pulsed bias with $\sim 3 \mathrm{~ms}$ duration time is imposed on the obstacle dur-

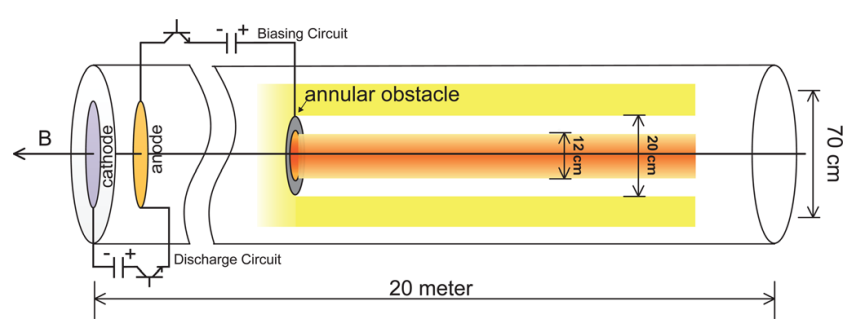

FIG. 1. (Color online) Schematic of LAPD including the annular obstacle and the biasing circuit. The annular obstacle blocks the ionizing fast electrons generated during the cathode-anode discharge and forms a plasma column with $12 \mathrm{~cm}$ diameter downstream. ing the steady portion of the LAPD discharge. The biasing voltage in this experiment is up to $250 \mathrm{~V}$, and it establishes radial electric fields and spins the edge of the $12 \mathrm{~cm}$-diameter plasma column through $E \times B$ drift. Figure 2 shows typical profiles of the plasma density $n_{e}$, root-mean-square (rms) of ion saturation current $\left(\mathrm{I}_{\mathrm{sat}}\right)$ fluctuation, and the plasma potential $\mathrm{V}_{\mathrm{p}}$, measured by Langmuir probes (described below) 0.3 $\mathrm{m}$ downstream from the annulus when the bias voltage is 100 V. Steep radial density and potential gradients are both localized at the inner edge of the obstacle $(\mathrm{r} \sim 6 \mathrm{~cm})$, along with large $I_{\text {sat }}$ fluctuation power. In the region within $\mathrm{r}<6 \mathrm{~cm}$, the applied radial electric fields are shorted by the fast electron population, which ties the local plasma potential to the plasma

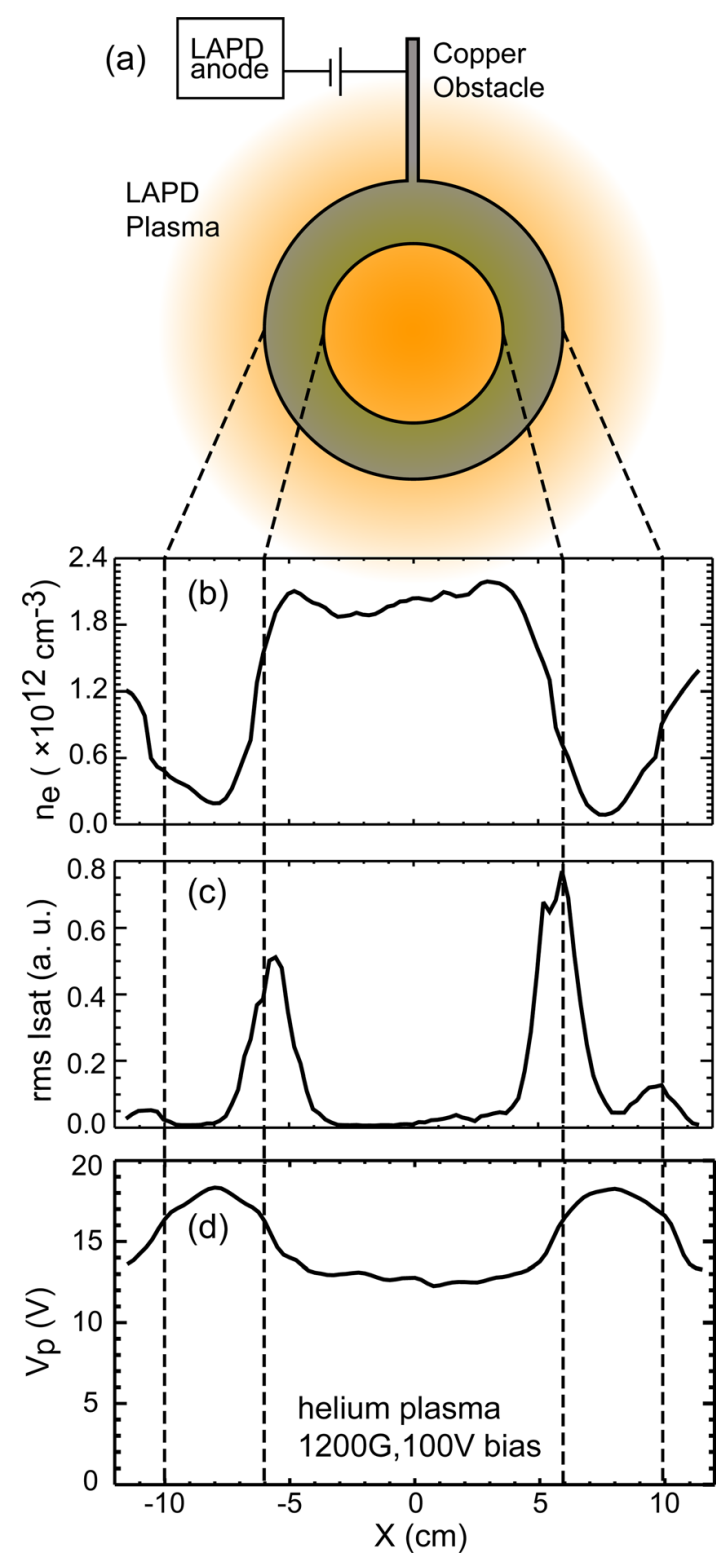

FIG. 2. (Color online) (a) Schematic of the LAPD end-view. The copper obstacle can be biased relative to the LAPD anode by an external power supply. (b) Typical helium plasma density profile. (c) Typical root-mean-square (RMS) fluctuation of the ion saturation current. (d) Plasma potential profile $0.3 \mathrm{~m}$ downstream from the obstacle when the obstacle is biased to $100 \mathrm{~V}$ in helium plasma. 


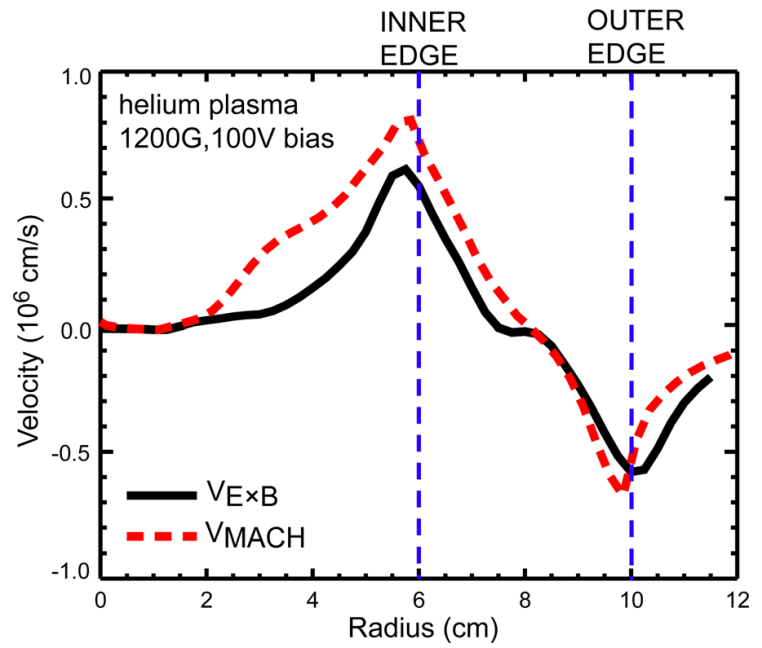

FIG. 3. (Color online) Profile of plasma flow velocity measured by the Mach probe (dashed line) and the $E \times B$ drift velocity profile (solid line) calculated from the plasma potential $\left(\mathrm{V}_{\mathrm{p}}\right)$ measurement of a swept Langmuir probe.

source. Thus the effects of applied electric fields and sheared $E \times B$ flow are limited to the obstacle edge regions.

Measurements of density, potential, temperature, and their fluctuations are made using Langmuir probes. A 3-tip probe is used as a triple Langmuir probe ${ }^{15}$ to measure the fluctuations of ion saturation current $\left(\mathrm{I}_{\mathrm{sat}} \propto n_{e} \sqrt{T_{e}}\right)$, floating potential $\left(V_{f}\right)$ and electron temperature $\left(T_{e}\right)$. A $65 \mathrm{~V}$ bias is applied between two tips measuring $\mathrm{I}_{\mathrm{sat}}$ to reject the primary electrons. A single-tip probe is also used as a swept Lang- muir probe to measure the equilibrium profiles of plasma potential $\left(\mathrm{V}_{\mathrm{p}}\right)$ and electron temperature. Two different methods are used to quantify the sheared flow profile. Firstly, the $E \times B$ drift velocity is calculated from the plasma potential profile measured by a swept Langmuir probe. Then, a Gundestrup (Mach) probe ${ }^{16}$ with six faces is used to measure the flows directly in several bias voltages. Figure 3 shows the radial profiles of the flow velocity measured by both methods in helium plasma with $150 \mathrm{~V}$ bias on the annulus. The flow velocity peaks at $\mathrm{r}=6 \mathrm{~cm}$ and $10 \mathrm{~cm}$, which aligns to the inner and outer edge of the annular obstacle. The peak velocity is comparable to the ion sound speed $v_{s}$ with Mach number $\sim 1\left(v_{s} \sim \sqrt{K T_{e} / M_{i}} \sim 1.1 \times 10^{6} \mathrm{~cm} / \mathrm{s}\right)$. The velocity profile measured by the Mach probe agrees the calculated $E \times B$ drift. And the $E \times B$ drift velocity is much larger than the electron diamagnetic drift velocity $\left(v_{D e}=\left(k T_{e} / e B_{z}\right)\left(n_{0}^{\prime} / n_{0}\right) \sim 2 \times 10^{5} \mathrm{~cm} / \mathrm{s}\right)$. This suggests that the $E \times B$ drift dominates the plasma edge flow dynamics. The fluctuations of the magnetic fields are measured by a B-dot probe. A typical magnetic fluctuation level observed is $\delta B / B_{z} \leq 0.1 \%$, so the electrostatic approximation is assumed in all of the analysis below.

The relation between the external bias applied to the obstacle and the plasma potential $\left(\mathrm{V}_{\mathrm{p}}\right)$ formed downstream of the obstacle is shown in Fig. 4(a), measured in neon plasmas with $B_{z}=1200 \mathrm{G}$. The plasma potential profiles are measured by a swept Langmuir probe located $0.3 \mathrm{~m}$ downstream of the obstacle. Then the $\mathrm{V}_{\mathrm{p}}$ profiles are fit by a function of the form $A_{2}+\left(A_{1}-A_{2}\right) /\left(1+e^{\left(x-x_{0}\right) / d x}\right)$, where $A_{1}, A_{2}, x_{0}$, and $d x$ are constants. The azimuthal $E \times B$ velocity and the shearing rate are derived from gradients of the fitted potential
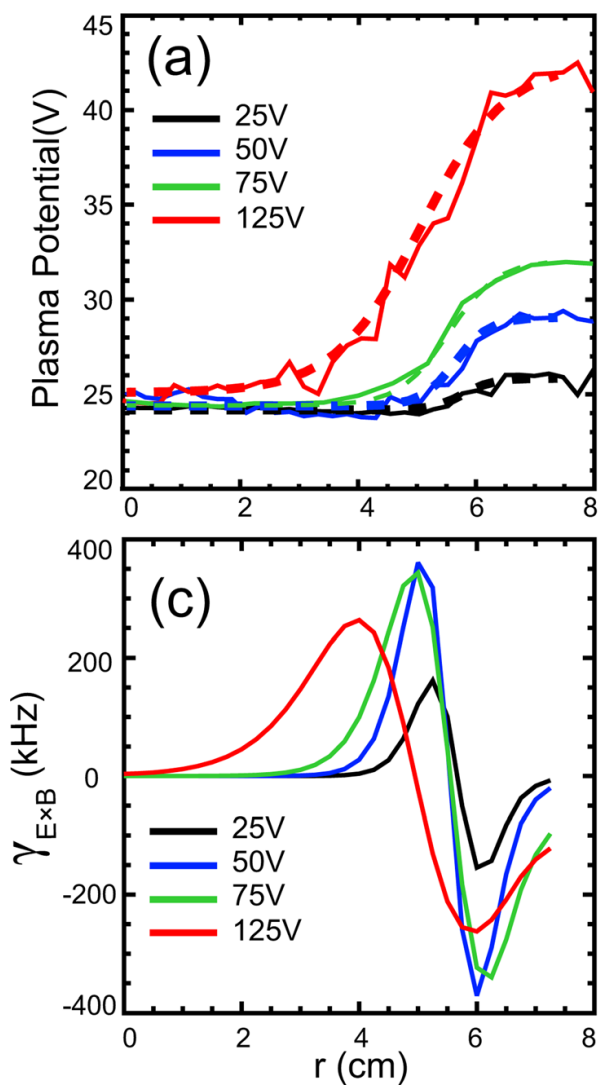
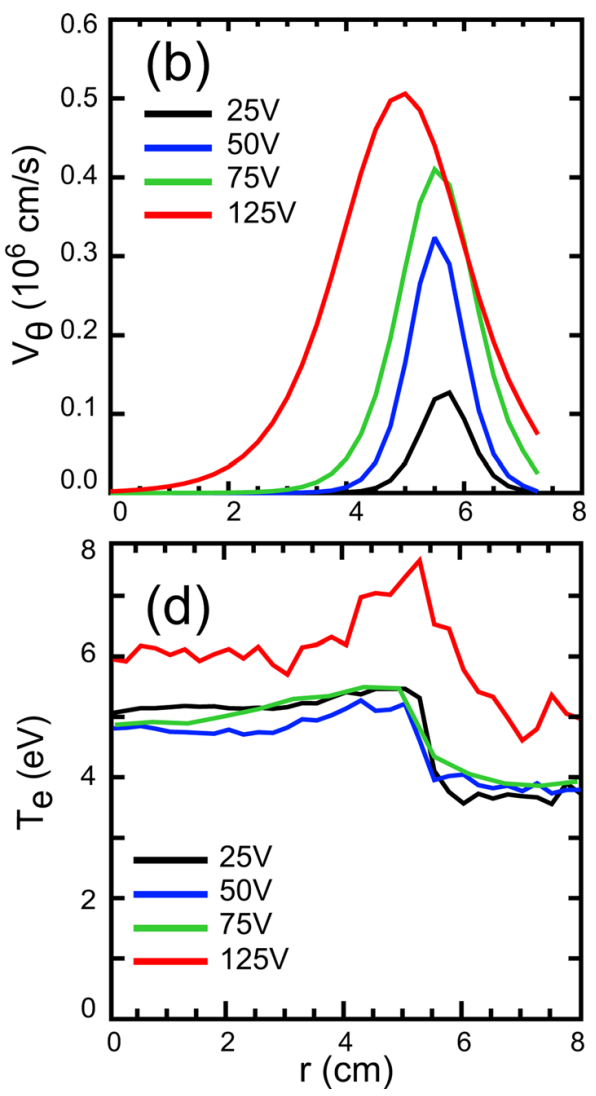

FIG. 4. (Color online) Profiles of plasma potential with fit $\left(\mathrm{V}_{\mathrm{p}}\right)$, azimuthal $E \times B$ drift velocity $\left(v_{\theta}\right)$, shearing rate $\left(\gamma_{E \times B}\right)$ and electron temperature $\left(T_{e}\right)$ for several bias values (neon plasma, $B_{z}=1200 \mathrm{G}$ ). Measurement of $\mathrm{V}_{\mathrm{p}}$ and $T_{e}$ are made by a swept Langmuir probe, while $v_{\theta}$ and $\gamma_{E \times B}$ are calculated based on the fitted $V_{p}$ profiles. 
profiles. Profiles of the azimuthal velocity $v_{\theta}$ and the shearing rate $\gamma$ (defined as $\gamma=\partial v_{\theta} / \partial r$ ) are shown in Figs. 4(b) and 4(c), with electron temperature profiles in Fig. 4(d). The peak plasma potential and the azimuthal drift velocity both increase with the bias on the obstacle. However, the shearing rate peaks at $50 \mathrm{~V}-75 \mathrm{~V}$ bias, while with $125 \mathrm{~V}$ bias, the maximum of the shearing rate shifts radially inward significantly. The $T_{e}$ profile is approximately constant with bias up to $75 \mathrm{~V}$, and increases by $\sim 20 \%$ with $125 \mathrm{~V}$ bias.

\section{EXPERIMENTAL RESULTS}

\section{A. Overview}

In this experiment, the plasma edge turbulence and the associated turbulent transport are modified in three different ways: (a) alter the bias voltage $V_{\text {bias }}$ that is applied on the annular obstacle $(0 \mathrm{~V}-250 \mathrm{~V})$, (b) change the axial magnetic field strength $B_{z}(600 \mathrm{G}-1800 \mathrm{G})$, and (c) switch the plasma species (helium or neon). A previous study on the modification of the LAPD edge turbulence by biasing the vacuum vessel $^{8}$ shows that the amplitude and shearing rate of the edge flow are crucial parameters in the confinement transition. Studies of the gradient driven instabilities (e.g., the discussion of fig. 4 in Ref. 17) usually suggest that the scale size of the turbulent structure is proportional to the ion sound radius $\rho_{s}$. So, in this experiment, it is expected that the edge turbulence and transport are associated with both the azimuthal flow induced by the $E \times B$ drift and the value of $\rho_{s}$. Figure 5 plots all the cases studied in this experiment in term of the values of $\left(V_{\text {bias }} / B_{z}\right)$ and $\left(\rho_{s}^{-1}\right)$, with control parameters (plasma species, $V_{\text {bias }}$ and $B_{z}$ ) noted. The observed plasma characteristics can be categorized into two very different

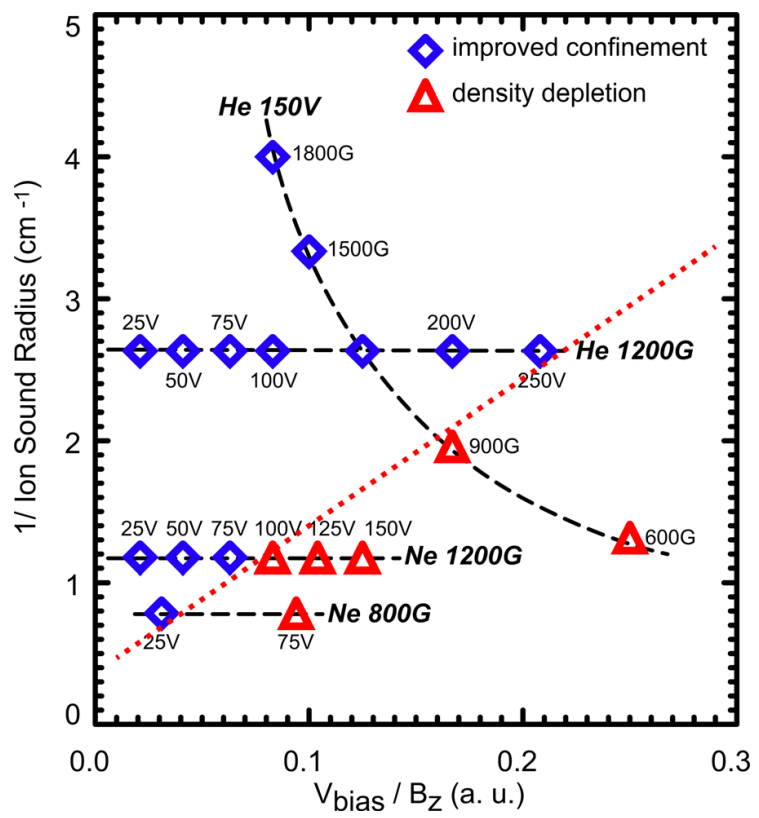

FIG. 5. (Color online) Plot of all cases studied with control parameters marked. Horizontal axis indicates the direction of increasing flow velocity; vertical axis indicates the direction of decreasing ion sound radius $\left(\rho_{s}\right)$. Dotted line is drawn to guide the eye. Cases with large radial transport and plasma depletion tend to be associated with large flow velocity and large $\rho_{s}$. states: enhanced density confinement (blue diamonds) and density depletion (red triangles). Typical spatial-temporal evolutions of $\mathrm{I}_{\mathrm{sat}}$ signals for both states are shown in Figs. 6(a) and 6(b). Comparison of plasma density profile $\left(n_{e} \propto \mathrm{I}_{\mathrm{sat}} / \sqrt{T_{e}}\right)$ before the end of the pulsed bias in these cases are shown in Fig. 7(a). The plasma characteristics when no bias is applied to the obstacle (floating obstacle) is usually used for comparison. In cases with improved confinement [Fig. 6(a)], a much steeper edge density gradient, associated with increasing plasma density within the plasma column is observed. In helium plasma, the density growth is more significant at the edge region of the column; while in neon plasma, the density growth is larger near the center region. In cases with "density depletion" [Fig. 6(b)], the density of the plasma column transiently increases in the central region upon the application of the bias, then decreases rapidly during the remainder of the biasing pulse, becoming totally depleted in some cases. Meanwhile, the density downstream of the obstacle outer edge ( $r \geq 10 \mathrm{~cm}$ region)
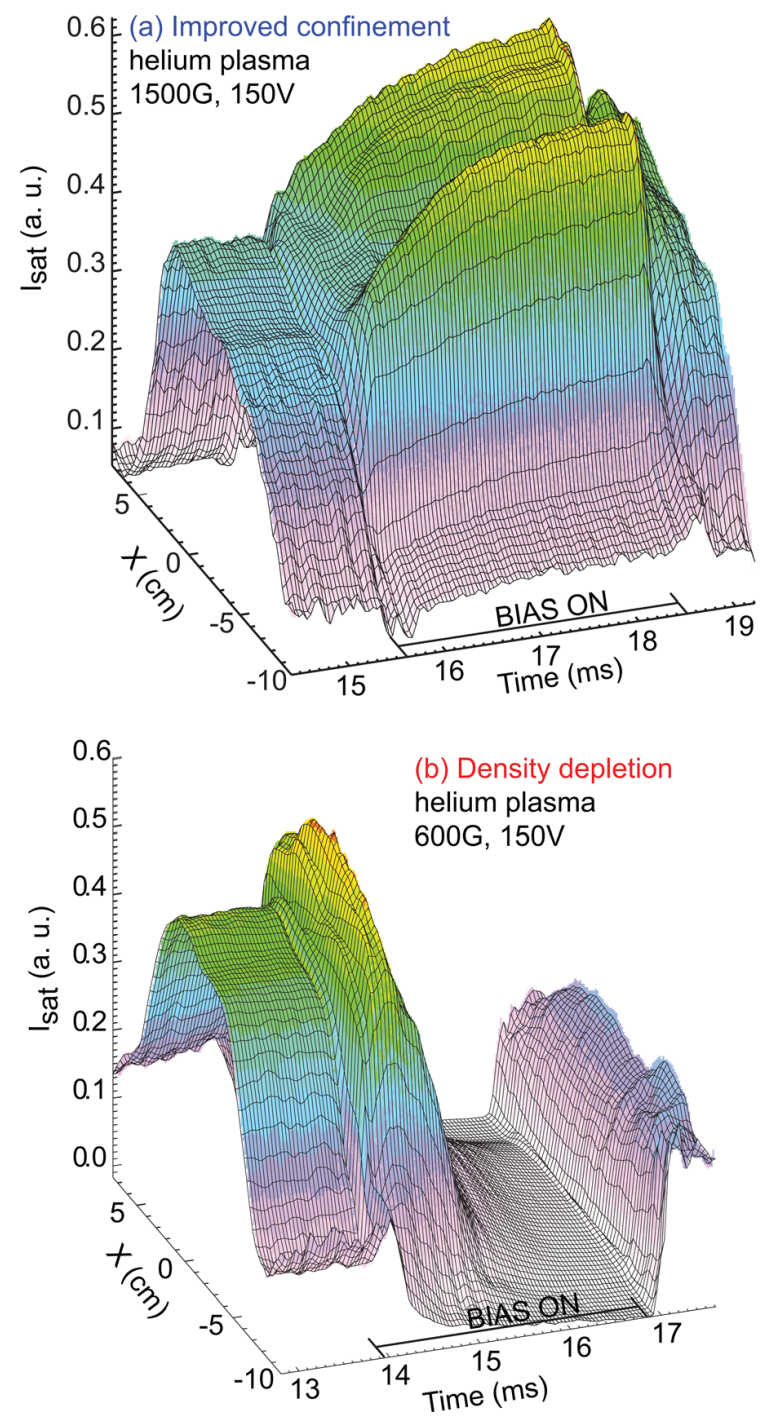

FIG. 6. (Color online) Space and time resolved ion saturation current ( $\left.\mathrm{I}_{\text {sat }}\right)$ profiles in (a) a case of improved confinement and (b) a case of plasma depletion. 

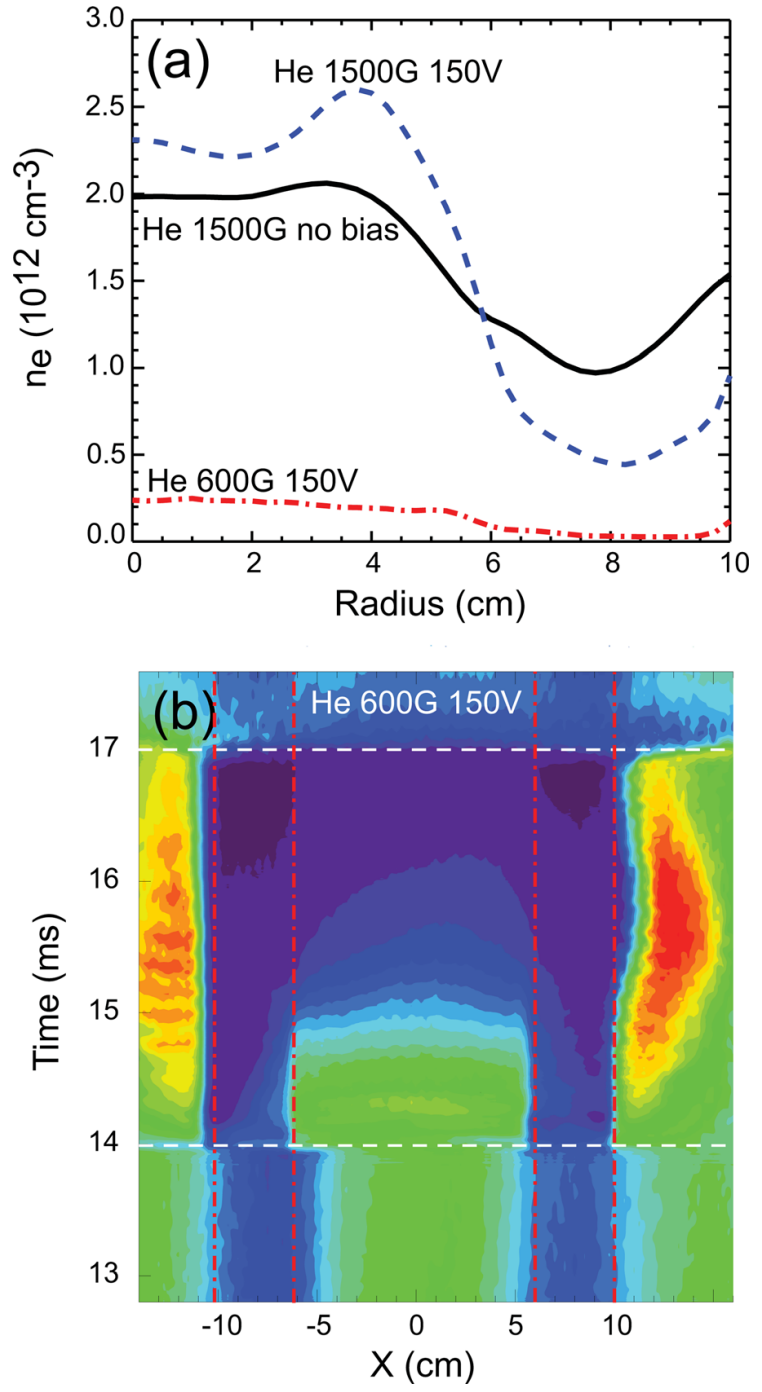

FIG. 7. (Color online) (a) Density profiles $\left(n_{e} \tilde{I}_{\mathrm{sat}} / \sqrt{T_{e}}\right)$ in cases with improved confinement (dashed line), plasma depletion (dashed-dotted line), and when the obstacle is floating (solid line), with control parameters marked. (b) The contour of time and space resolved $I_{\text {sat }}$ signal in helium plasma with $B_{z}=600 G$ and $V_{\text {bias }}=150 \mathrm{~V}$. The positions of the inner and outer radius of the obstacle is marked (dashed-dotted lines). Outward density transport is indicated by the simultaneous evolution of the $I_{\text {sat }}$ signal at the inner plasma column and the outer-edge plasma.

increases during the bias pulse, with the time sequence correlated to the depletion of the inner plasma column [Fig. 7(b)]. This is observed in all cases with density depletion, suggesting that large cross-field transport happens in the radial direction during the biasing time. Overall, enhanced confinement cases are usually associated with smaller $\rho_{s}$ and slower edge $E \times B$ flow velocity (upper-left in Fig. 5), while density depletion cases are associated with larger $\rho_{s}$ and faster edge $E \times B$ flow (lower-right in Fig. 5).

The density evolutions during the bias pulse for both states are also suggested by the high-speed camera imaging data (Fig. 8), which observes the visible light intensity from the end-window of the LAPD. Typical cases with improved confinement or density depletion are investigated. The evolution of the light intensity observed after the pulsed bias is applied (at $\sim 0 \mathrm{~ms}$ in Fig. 8 ) agrees with the $\mathrm{I}_{\text {sat }}$ data. (a) helium 150V 1200G

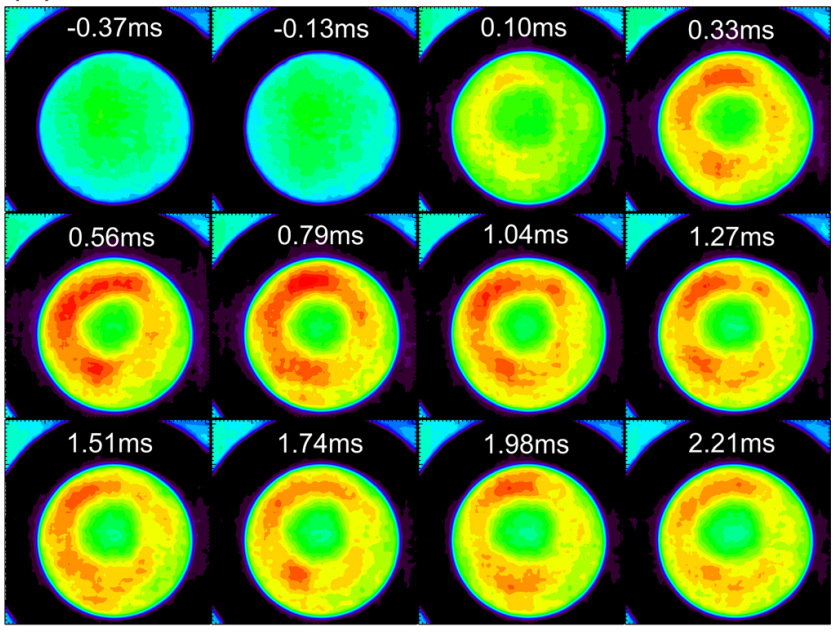

(b) helium 150V 600G

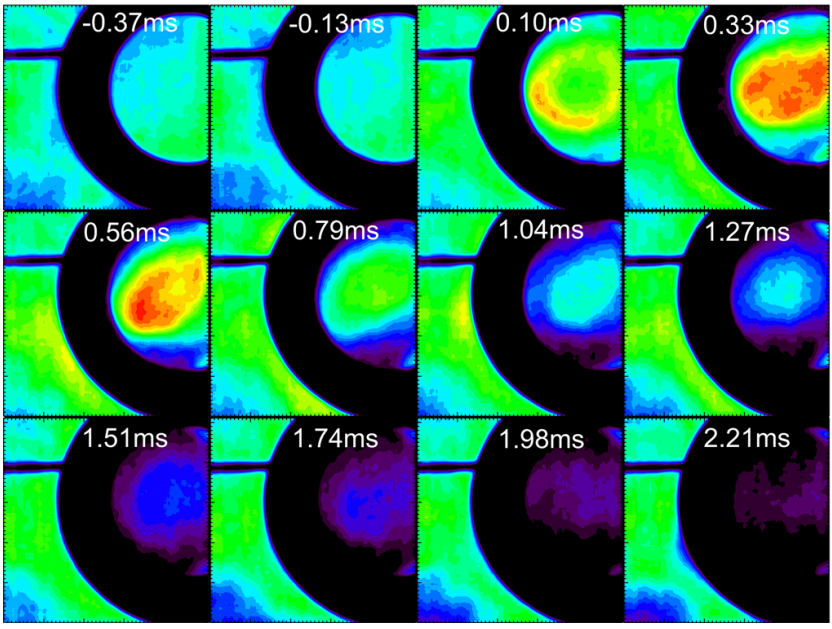

FIG. 8. (Color online) Intensity of the visible light measured by the highspeed camera imaging for (a) improved confinement and (b) density depletion. The camera is located at the north end-window of LAPD and facing the cathode. The black annular region suggests the shadow of the obstacle. The obstacle bias pulse starts at $0 \mathrm{~ms}$.

\section{B. Confinement transition and radial flux}

To understand the transition from improved confinement to depletion of the plasma density, three sets of data are analyzed in detail: (a) in helium plasma with $B_{z}=1200 \mathrm{G}$ and $V_{\text {bias }}=25 \mathrm{~V}-250 \mathrm{~V}$; (b) in neon plasma with $B_{z}=1200 \mathrm{G}$ and $V_{\text {bias }}=25 \mathrm{~V}-150 \mathrm{~V}$; (c) in helium plasma with $V_{\text {bias }}=150 \mathrm{~V}$ and $B_{z}=600 \mathrm{G}-1800 \mathrm{G}$. To quantify the density evolution when sweeping the bias or the axial magnetic field, the averaged densities at the center region of the plasma column $(r \leq 2 \mathrm{~cm})$ are calculated from the $\mathrm{I}_{\text {sat }}$ measurement. Figure 9 shows the plasma densities $\bar{n}_{e, r \leq 2 \mathrm{~cm}}$, normalized to the density when no bias is applied on the annular obstacle. The no-bias case is used as a reference to minimize the uncertainty induced by changes in machine condition (such as the discharge power and the cathode condition) for data acquired at different run times. It is observed that in helium plasmas with $B_{z}=1200 \mathrm{G}$ [Fig. 9(a)], the central density linearly increases with the applied bias. A similar density increase is also initially observed in neon plasmas, 

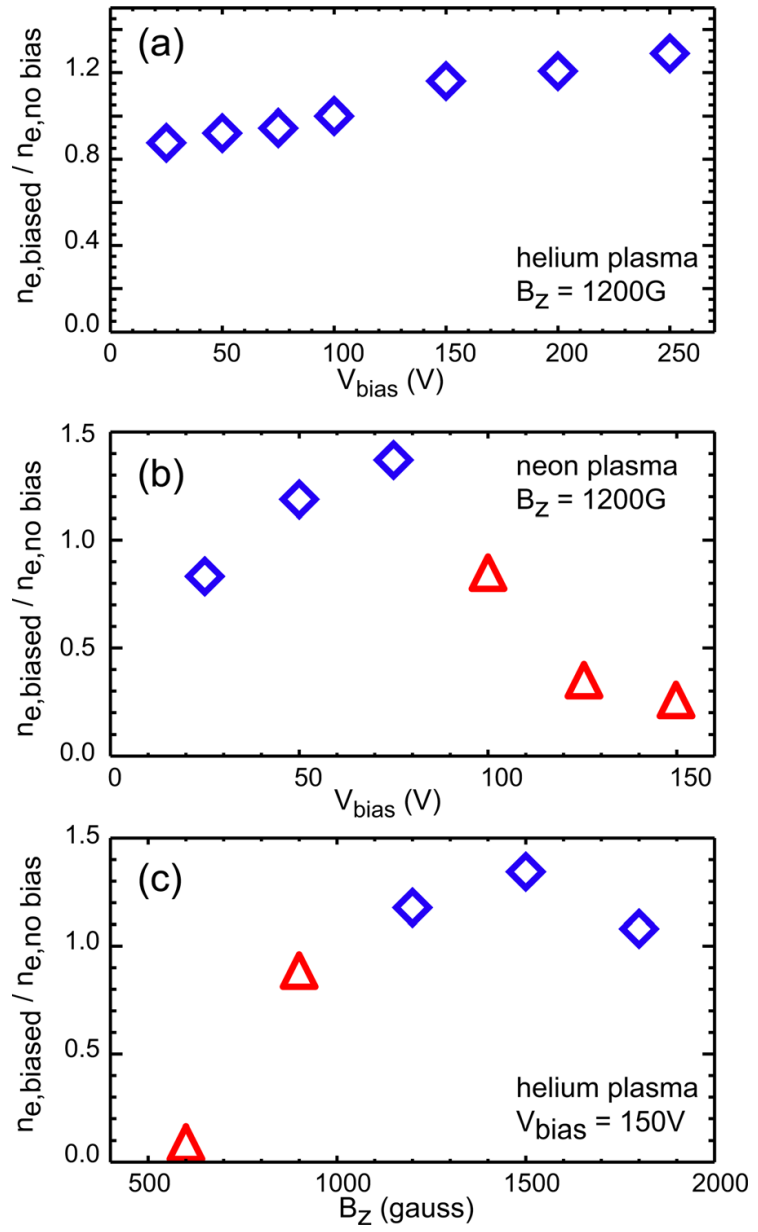

FIG. 9. (Color online) Evolution of the averaged density at the center of the plasma column $(r \leq 2 \mathrm{~cm})$ with increased bias voltage on the obstacle $\left(V_{\text {bias }}\right)$ or axial magnetic field $\left(B_{z}\right)$. The densities are normalized to the averaged plasma density when the obstacle is floating. Diamonds represent cases with improved confinement, while triangles represent cases with plasma depletion.

until the transition to large outward transport occurs at $V_{\text {bias }} \sim 100 \mathrm{~V}$ [Fig. 9(b)]. The confinement transition correlates with the scale length of the ion sound gyroradius $\rho_{s}$. For the same edge biasing $\left(V_{\text {bias }} \geq 100 \mathrm{~V}\right)$ and field
$\left(B_{z}=1200 \mathrm{G}\right)$, the confinement transition happens in neon plasma (with $\rho_{s}=0.85 \mathrm{~cm}$ ), but not in helium plasma (with $\left.\rho_{s}=0.38 \mathrm{~cm}\right)$. In helium plasmas, the confinement transition happens when $B_{z} \leq 900 \mathrm{G}$ [Fig. $9(\mathrm{c}), \rho_{s} \geq 0.51 \mathrm{~cm}$ ].

Direct evaluations of the radial particle flux are made using the $\tilde{\mathrm{I}}_{\mathrm{sat}}$ and the floating potential $\tilde{\mathrm{V}}_{\mathrm{f}}$ data measured simultaneously by triple probes. The electrostatically driven particle flux is calculated by

$$
\begin{aligned}
\Gamma_{n} & =\left\langle n v_{r}\right\rangle=\frac{\left\langle n E_{\theta}\right\rangle}{B_{z}} \\
& =\frac{2}{B} \int_{0}^{\infty}|n(\omega)|\left|E_{\theta}(\omega)\right| \gamma_{n, E_{\theta}}(\omega) \cos \left(\theta_{n, \mathrm{E}_{\theta}}(\omega)\right) d \omega,
\end{aligned}
$$

where $n(\omega)$ and $E_{\theta}(\omega)$ are the FFT density and azimuthal electric field, $\gamma_{n, E_{\theta}}(\omega)$ and $\theta_{n, E_{\theta}}(\omega)$ are the cross-coherency and cross-phase between $n(\omega)$ and $E_{\theta}(\omega)$. The fluctuation of the azimuthal electric field is not directly measured in this experiment. Instead, an estimate of $\left|E_{\theta}(\omega)\right|$ is made using the fluctuation of the floating potential $\tilde{\mathrm{V}}_{\mathrm{f}}$. Assuming the waves at the edge plasma have a drift-wave like dispersion relation $\left(k_{\theta} \sim \omega\right)$ and that the electron temperature fluctuation $\tilde{T}_{e} / T_{e}$ is much smaller than the density fluctuation $\tilde{n}_{e} / n_{e}$, one can estimate the four terms in Eq. (1) by

$$
\begin{gathered}
\left|\tilde{\mathrm{I}}_{\mathrm{sat}}\right| \propto\left|\tilde{n}_{e} \sqrt{T_{e}}\right| \propto\left|\tilde{n}_{e}\right| \\
\left|\tilde{E}_{\theta}(\omega)\right| \sim\left|-\nabla_{\theta} \tilde{\phi}(\omega)\right| \sim\left|k_{\theta} \tilde{\phi}(\omega)\right| \sim\left|\frac{\omega}{v_{D}} \tilde{\phi}(\omega)\right| \\
\propto|\omega \cdot \tilde{\phi}(\omega)|, \\
\gamma_{n, E_{\theta}}(\omega) \simeq \gamma_{n, \phi}(\omega), \\
\cos \left(\theta_{n, E_{\theta}}(\omega)\right) \sim \cos \left(\theta_{n, \phi}(\omega)-\frac{\pi}{2}\right) \sim \sin \left(\theta_{n, \phi}(\omega)\right) .
\end{gathered}
$$

Then the radial particle flux profile $\Gamma_{n}(r)$ can be calculated. Figure 10(a) shows the evolution of the radial flux profiles as a function of the bias on the obstacle. Positive flux indicates radially outward transport and negative flux indicates inward transport. It is known from Eq. (1) that the direction of the (a) neon, $1200 \mathrm{G}$

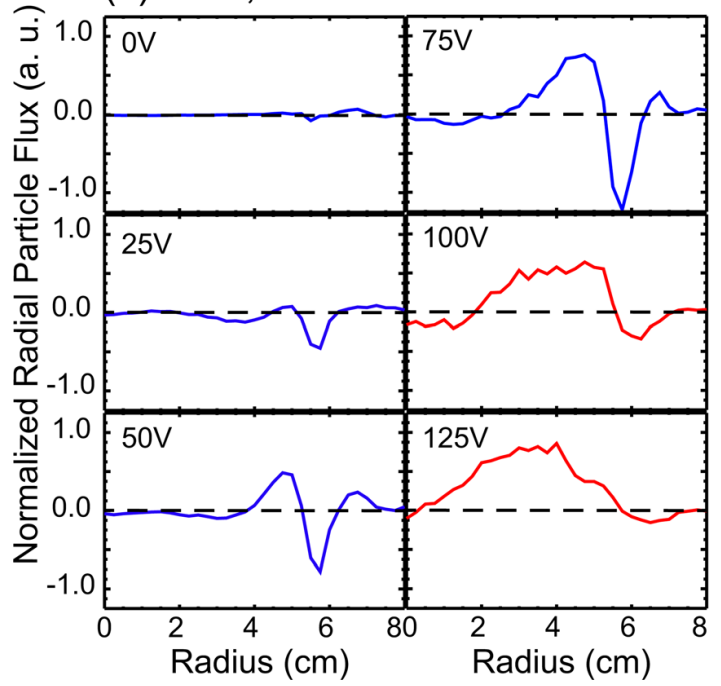

\section{(b) helium, 150V bias}

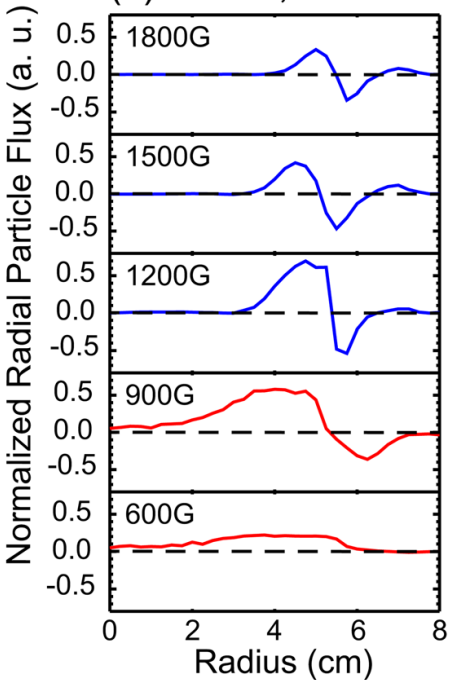

FIG. 10. (Color online) (a) Radial particle flux profiles with increasing bias on the obstacle in neon plasma with $B_{z}=1200 \mathrm{G}$. Positive flux represents radially outward. (b) Radial particle flux profiles with various $B_{z}$ in helium plasma with $150 \mathrm{~V}$ bias on the obstacle. All profiles are averaged over the $\sim 3 \mathrm{~ms}$ bias time. 
radial flux only depends on the sign of $\sin \left(\theta_{n, \phi}(\omega)\right)$, which suggests that the cross-phase between the density and potential structures is very important in the radial transport. When increasing the bias on the obstacle from $0 \mathrm{~V}$ to $75 \mathrm{~V}$ (the cases with improved confinement), a sheared radial flux profile is observed at the edge of the plasma column, where the maximum flow shear is localized. Outward radial flux is observed at the inner region of the plasma edge. However, a steep change of the radial flux profile, with an inward flux layer at the outer region of the plasma edge, is formed. The reversed flux profile across the radius leads to steeper edge density gradient and improved plasma confinement. The amplitude of the outward and inward flux both increase with the bias voltage, indicating that the sheared azimuthal flow plays an important role in the formation of the flux reversal. Similar flux profiles are observed in the edge plasmas of tokamaks (e.g., in TEXTOR (Ref. 18)) and basic devices (e.g., in CSDX (Ref. 19)), where sheared edge flows are driven either by applied external radial electric fields or by the selfgenerated nonlinear zonal flow. However, when the bias is further increased ( $V_{\text {bias }} \geq 100 \mathrm{~V}$, cases with density depletion), the outward particle transport dominates the radial flux profiles, and the radial width of the outward flux is comparable to the radius of the plasma column. These results agree well with the observed density evolution within the plasma column in all the cases. The evolution of the radial flux with modified $B_{z}$ is shown in Figure $10(\mathrm{~b}) . B_{z}$ is also an effective control parameter to modify the flow velocity. In addition, it affects the turbulent waves by modifying the scale length of $\rho_{s}$. The radial particle flux profiles are similar to that with $V_{\text {bias }}$ modified. Strong axial magnetic field is favorable to improved plasma confinement, while weak field leads to large outward transport and plasma depletion. This is also consistent with the observed density profiles in these cases.

\section{Spectrum and correlation profiles of the edge turbulence}

Having examined the radial flux profiles of the cases with both improved confinement and density depletion, we now turn to the plasma edge fluctuations that account for the density behavior. Figure 11 shows the radial profiles of the $\mathrm{I}_{\text {sat }}$ fluctuating power during the 3-ms pulsed bias on the obstacle, integrated over all frequencies and normalized to the maximum fluctuating power. It is observed that the radial location and extent of the fluctuating power is closely associated with the turbulent transport. Under larger $B_{z}$ (lower $V_{E \times B}$ and smaller $\left.\rho_{s}\right)$, the density fluctuation is localized at the edge region of the plasma column $(r \sim 6 \mathrm{~cm})$ and has a radial extent of $\sim 2 \mathrm{~cm}$ [Fig. 11(a)]. With the axial magnetic field lowered to $B_{z} \leq 900 \mathrm{G}$, the fluctuations extend throughout the entire plasma column. The lowered total power in cases with small $B_{z}$ is mainly the result of the rapid density decline during the pulse time, rather than the suppression of the turbulence. Similarly, when the obstacle bias is modified [Fig. 11(b)], in the cases with improved confinement $\left(V_{\text {bias }}=0 V-75 \mathrm{~V}\right)$, the fluctuations are localized at the edge region while, in the cases with density depletion $\left(V_{\text {bias }} \geq 100 \mathrm{~V}\right)$, the radial span of the fluctuation power profile is comparable to the radius of the plasma column. These fluctuation profiles suggest that electrostatic turbulent transport causes the steepening and flattening of the density profile that was described in Sec. III B.

Figure 12 presents space and frequency resolved power spectra for six bias voltages (a-f), and the two-dimensional cross-field correlation function of $\mathrm{I}_{\text {sat }}$ fluctuations under $75 \mathrm{~V}$ and $125 \mathrm{~V}$ bias $(\mathrm{g}-\mathrm{h})$ in neon plasmas. The frequency range of the edge fluctuations is $\sim 0-30 \mathrm{kHz}$ (the ion cyclotron frequency in this experiment is $f_{c i}=457 \mathrm{kHz}$ in helium plasma and $f_{c i}=91 \mathrm{kHz}$ in neon plasma with $\left.B_{z}=1200 \mathrm{G}\right)$. The frequency gaps between adjacent peaks in the spectrum increase with biasing voltage, indicating the effect of the Doppler-shift in frequency due to larger flow velocity. The two-dimensional (2D) correlation profiles are measured by two Langmuir probes, located downstream of the annulus and separated by $0.3 \mathrm{~m}$. The parallel wavelengths of the edge fluctuations are also measured using 2-point correlation techniques, with Langmuir probes separated by various distances. The observed parallel wavelength is $\lambda_{\|} \sim 15 \mathrm{~m}$, which is comparable to the machine length. Thus the $2 \mathrm{D}$ correlation measurements mostly reveal the mode structures perpendicular to the magnetic field. The correlation function with $75 \mathrm{~V}$ (a) helium, 150V bias

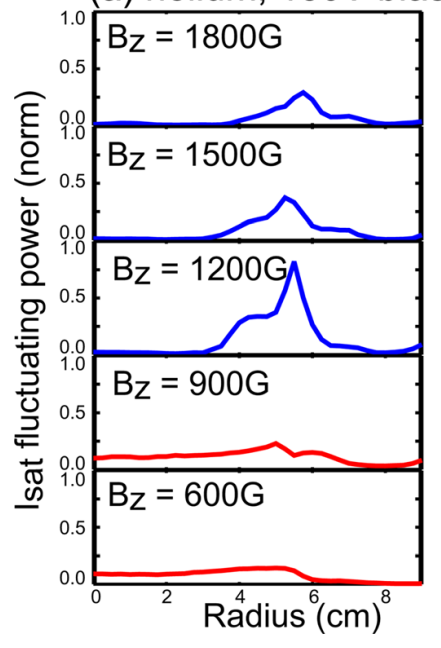

(b) neon, 1200G

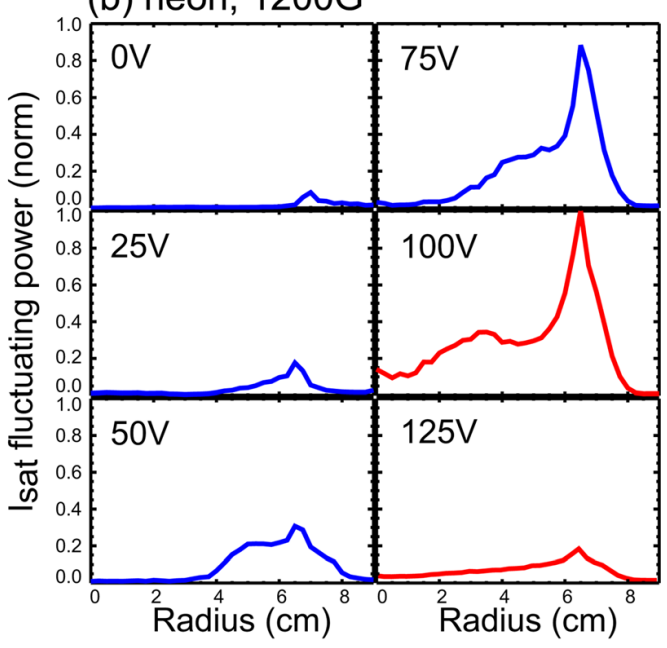

FIG. 11. (Color online) (a) Radial profiles of the fluctuating power of $I_{\text {sat }}$ (averaged over the 3-ms bias time and totaled over $2-100 \mathrm{kHz}$ ) with various $B_{z}$ in helium plasma with $150 \mathrm{~V}$ bias on the obstacle. Positive flux represents radially outward. (b) Radial profiles of the fluctuating power of $I_{\text {sat }}$ with increasing bias on the obstacle in neon plasma with $B_{z}=1200 G$. 

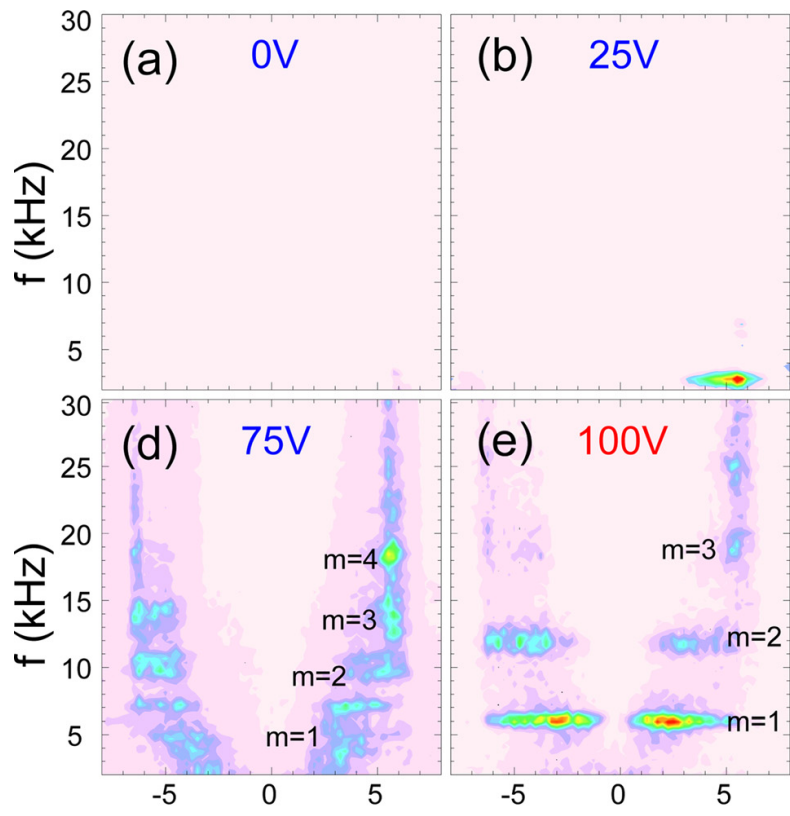

(e)

$100 \mathrm{~V}$
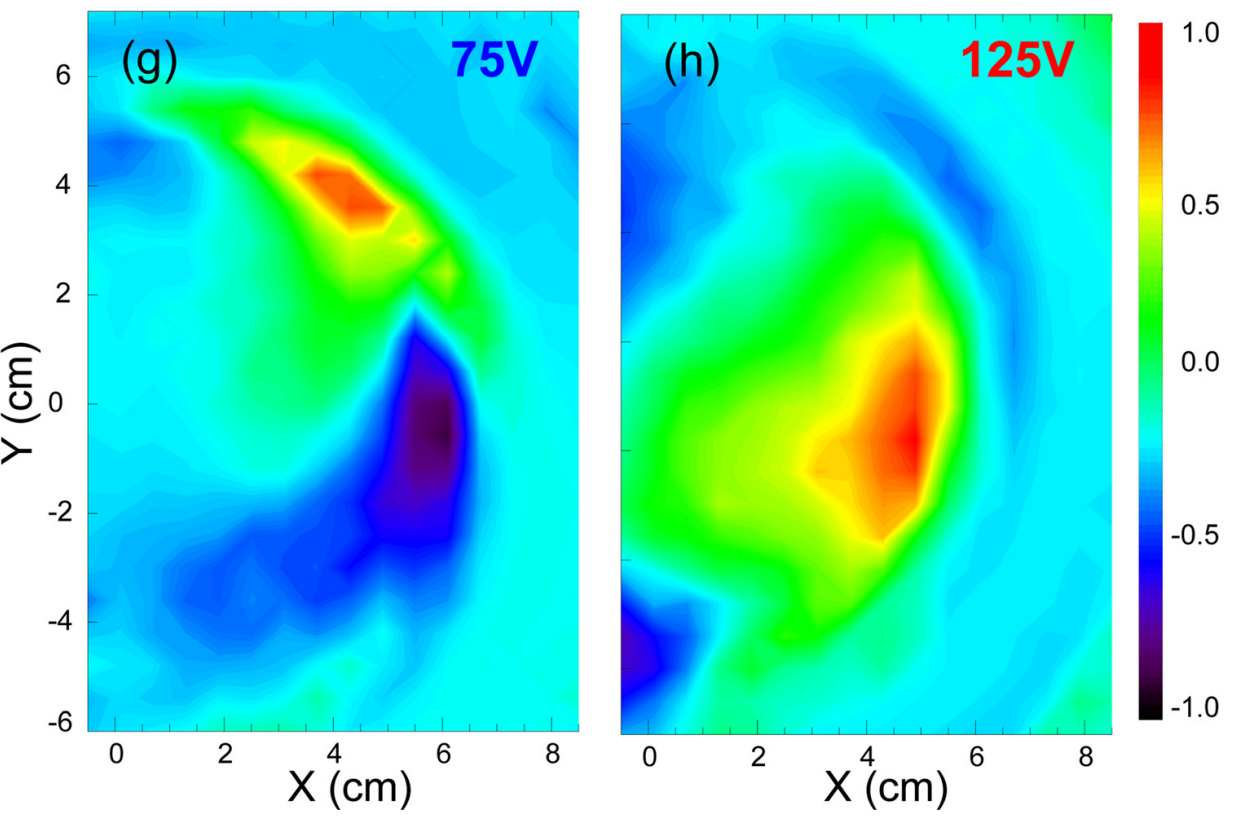

FIG. 12. (Color online) (a-f) Space resolved $I_{\text {sat }}$ FFT power spectra in neon plasma with $B_{z}=1200 G$ and increasing $V_{\text {bias. }}$. (g-h) Two-dimensional cross-field correlation functions for $I_{\text {sat }}$ fluctuations in cases (d) and (f). bias [Fig. 12(g)] indicates waves with an average mode number $\bar{m} \sim 2$. The correlated structures are asymmetric, elongated in the azimuthal direction. In contrast, with $125 \mathrm{~V}$ bias [Fig. 12(h)], an $m=1$ mode dominates. It suggests from the power spectrum that the mode numbers of the dominant modes decrease with increasing bias. The radial correlation length of the $m=1$ mode structure is $\sim 6 \mathrm{~cm}$, which is consistent with the observed fluctuating power and flux profile. Moreover, the $m=1$ mode is favorable for outward particle transport: the mode structure extends to the core region of the plasma column and large radial transport along the equipotential line is expected.

The correlation between density depletion and the $m=1$ mode is also evident by the time evolution of the fluctuation power spectrum at different bias. Figure 13 shows the time evolution of the ion saturation current $\left(I_{\text {sat }}\right)$ power spectrum for cases discussed above, calculated by Fast Fourier Trans- form (FFT) with moving time window. With $75 \mathrm{~V}$ bias on the annulus, the power spectrum is steady during the $\sim 3 \mathrm{~ms}$ pulse time, and modes with $m=1$ - 4 have comparable amplitude. With $100 \mathrm{~V}-150 \mathrm{~V}$ bias, damping of the modes are observed during the bias time, especially modes with higher $\mathrm{m}$ numbers. The up-chirping of the mode frequencies are usually observed in cases with density depletion. With decreasing plasma density during the bias time, the potential applied on the annular obstacle are less shielded by plasmas, which induces larger $E \times B$ drift velocity. This is reflected by a larger Doppler shift effect in frequency in the power spectrum, which explains the up-chirping of the mode frequencies. In all cases with density depletion, it is observed that the $m=1$ mode dominates in the power spectrum.

Similar dependencies on the control parameter are observed when the bias is kept constant and the axial magnetic field is varied (Fig. 14). With lower $B_{z}$, the edge 

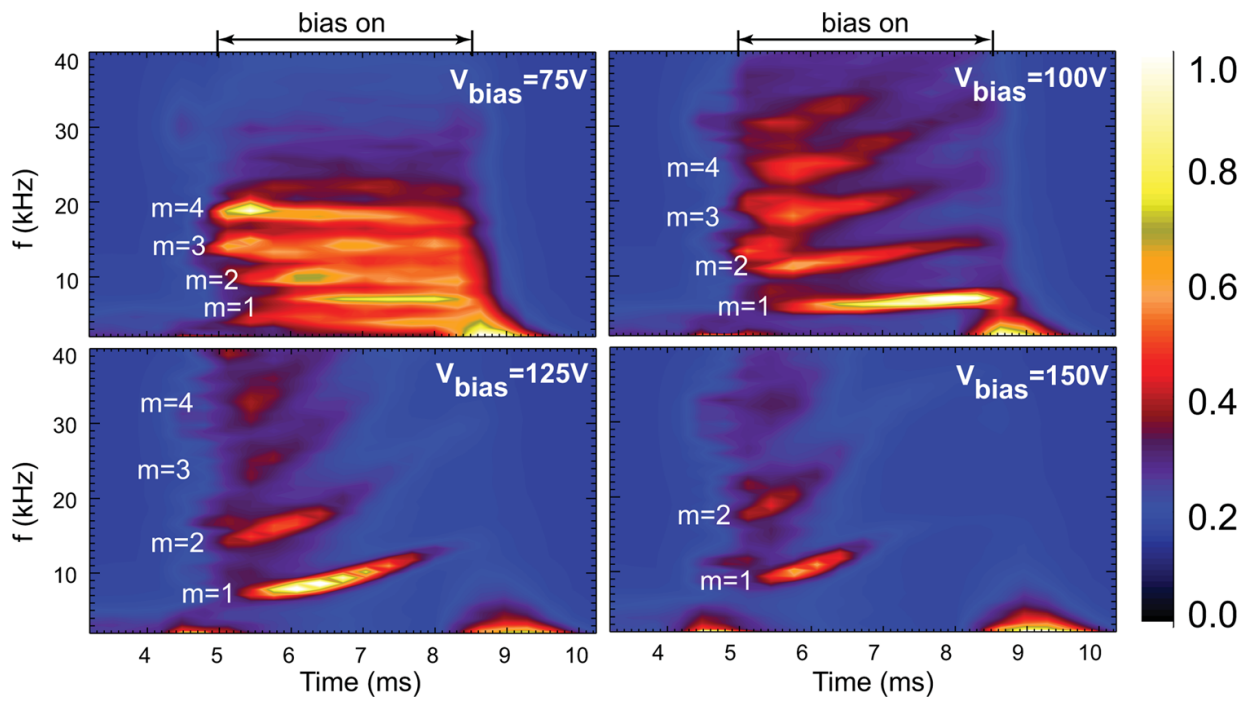

FIG. 13. (Color online) Time evolution of the power spectrum of fluctuations in ion saturation current for different bias voltage on the annulus. (a) $\mathrm{V}_{\text {bias }}=75 \mathrm{~V}$ (case with enhanced plasma confinement), (b)-(d) $\mathrm{V}_{\text {bias }}=100-150 \mathrm{~V}$ (cases with depletion in plasma density). fluctuations have smaller azimuthal mode numbers. Cases with density depletion are also associated with the $m=1$ mode and with large radial correlation length [Fig. 14(h)].

The radial flux profile changes sign at the plasma edge (Fig. 10). The mechanism responsible for this reversal is reversal of the cross-phase $\theta_{n, \phi}$ between the density and the potential structures. Figure 15 shows the 2-point cross-correlation functions of $\mathrm{I}_{\mathrm{sat}}$ (colored contour) and the floating potential $V_{f}$ (white dotted contour), measured using the same triple-probe-pair in helium plasma with $100 \mathrm{~V}$ bias at the obstacle (a case with improved confinement). The floating potential signal is adjusted according to the phase between the $\mathrm{I}_{\text {sat }}$ and $V_{f}$ signals at the reference probe, so that Fig. 15 reflect a snapshot of the density and potential structure with the right relative phase between them. The red dashed line indicates the inner edge of the obstacle, which also shows where the radial particle flux reverses its direction. Due to the sheared edge flow, the density and potential fluctuations develop radially sheared structures. The overlap between $\mathrm{I}_{\text {sat }}$ and $V_{f}$ structures suggests that a dramatic phase change between density and potential happens across the edge region. Opposite phases between $\mathrm{I}_{\text {sat }}$ and $V_{f}$ are observed at the inner and the outer region of the plasma edge (red dashed line). This result clearly confirms that changes in $\theta_{n, \phi}$ cause the observed reversal in radial particle flux.

\section{DISCUSSION AND LINEAR ANALYSIS OF THE EDGE MODES}

Identification of the wave modes of the observed edge turbulence is important in understanding the mechanisms of the particle flux and the effects of the biasing on the obstacle. The mode transition in a magnetized plasma column with sheared rotation has been discussed in several publications. ${ }^{9,20}$ In the current experiment, large local radial variation of the phase for plasma potential $\left(90^{\circ}-180^{\circ}\right.$, indicated in Fig. 15) is observed, which is a signature of the KelvinHelmholtz instability (as discussed in Ref. 20). However, the fact that the density and potential fluctuation has comparable fluctuation amplitude $\left(\delta n / n \sim e \phi / k T_{e} \sim 0.5\right)$ indicates that the density-gradient driven waves are also important in the edge fluctuation. In LAPD edge plasmas with radial electric field applied by edge biasing, a parameter regime where the turbulence is driven by both density gradient and sheared azimuthal flow is observed (so called Drift-Wave-KelvinHelmholtz (DWKH) regime, see Ref. 9). In DWKH regime, density and potential fluctuations are comparable, and the scale length of the radial velocity shear is comparable to the density gradient. In this experiment, the scale length of the density gradient $\left(L_{n} \sim 2 \mathrm{~cm}\right)$ and the velocity gradient $\left(L_{v}=\left|\ln v_{\theta}\right|^{-1} \sim 2 \mathrm{~cm}\right)$ are comparable to each other, which also fits with the DWKH parameter regime.

Linear analysis on the edge wave modes is done using a Braginskii two-fluid model. ${ }^{21}$ Although full nonlinear simulation might be necessary to compare with the experimental results, simple analytic solutions of the fluid equations using the experimental parameters help in mode identification. The fluid equations used here represent conservation of density, electron and ion momentum, and charge,

$$
\begin{aligned}
& (\partial \mathrm{t}+\mathrm{ve} \cdot \nabla) \mathrm{n}=0 \\
& \mathrm{~nm}_{\mathrm{e}}\left(\partial_{t}+\mathrm{v}_{\mathrm{e}} \cdot \nabla\right) \mathrm{v}_{\mathrm{e}}=-\nabla \mathrm{p}_{\mathrm{e}}-\mathrm{ne}\left(\mathrm{E}+\frac{1}{\mathrm{c}} \mathrm{v}_{\mathrm{e}} \times \mathrm{B}\right)-\mathrm{nm}_{\mathrm{e}} \nu_{\mathrm{ei}} \mathrm{v}_{\mathrm{e}} \\
& \mathrm{nm}_{\mathrm{i}}\left(\partial_{\mathrm{t}}+\mathrm{v}_{\mathrm{i}} \cdot \nabla\right) \mathrm{v}_{\mathrm{i}}=\mathrm{ne}\left(\mathrm{E}+\frac{1}{\mathrm{c}} \mathrm{v}_{\mathrm{i}} \times \mathrm{B}\right)-\mathrm{nm}_{\mathrm{i}} \nu_{\mathrm{in}} \mathrm{v}_{\mathrm{i}} \\
& \nabla \cdot \mathrm{J}=0, \quad \mathrm{~J}=\mathrm{en}\left(\mathrm{v}_{\mathrm{i} \|}-\mathrm{v}_{\mathrm{e} \|}\right)+\mathrm{en}\left(\mathrm{v}_{\mathrm{i} \perp}-\mathrm{v}_{\mathrm{e} \perp}\right) .
\end{aligned}
$$

A detailed description of the application of the two-fluid model on LAPD edge turbulence can be found in Ref. 12 .

Here we present the linear solutions of the fluid equations using experimentally obtained profiles of the plasma density and potential. The instabilities investigated are driftwave (DW), interchange instability (IC), and KelvinHelmholtz instability $(\mathrm{KH})$. Their frequencies and growth rates are compared. The density profile used in all cases is obtained in neon plasma with $75 \mathrm{~V}$ bias. Different potential profiles are used to model specific instabilities. The resistive drift wave branch is driven by the gradient of the density profile alone [Fig. 16(a)]. To model the interchange mode (IC) induced by the plasma rotation, a uniform-rotation potential profile $\phi_{0}(r)=\Omega r^{2} / 2$ is chosen [Fig. 16(b)] with 


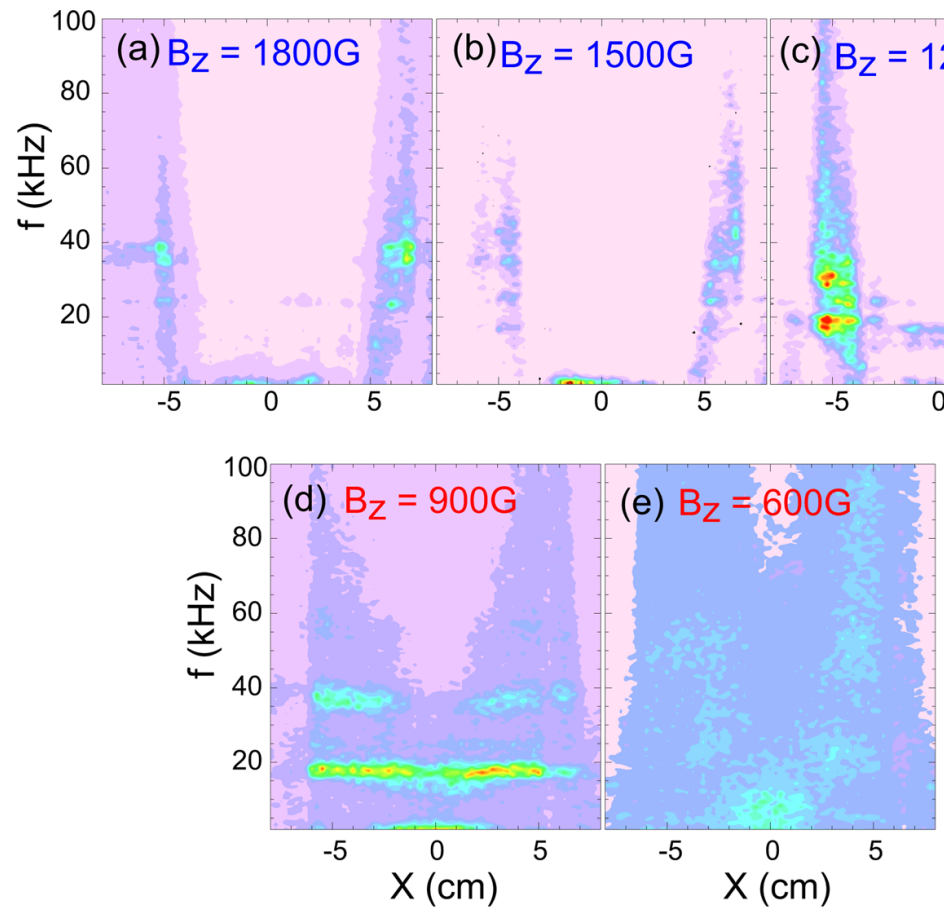

1.0

0.8

0.6

0.4

0.2

FIG. 14. (Color online) (a-e) Space resolved $I_{\text {sat }}$ FFT power spectra in helium plasma with $V_{\text {bias }}=150 \mathrm{~V}$ and decreasing $B_{z}$. (f-h) Two-dimensional cross-field correlation functions for $I_{s a}$ fluctuations in cases (a), (c), and (d).

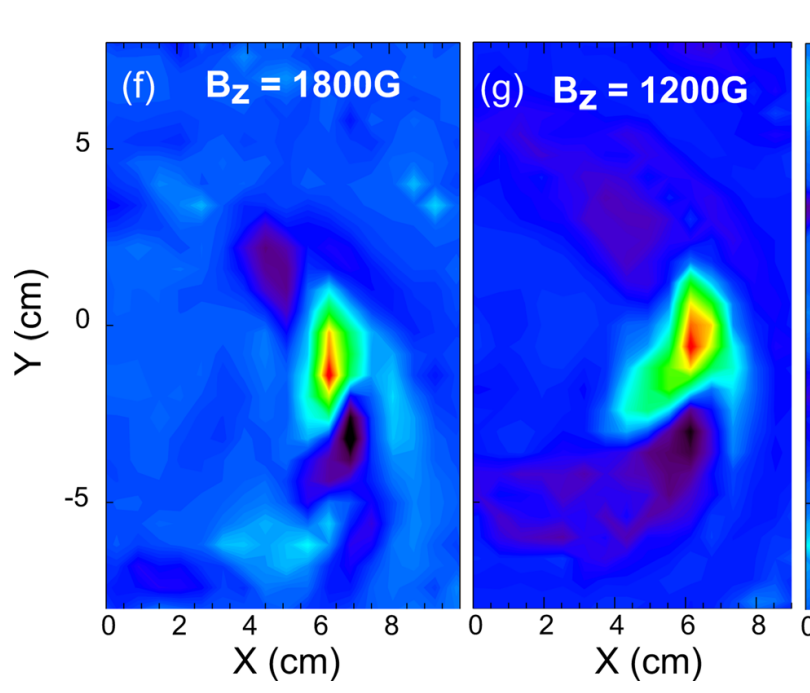

the rotation frequency comparable to the maximum rotation frequency in the experimental potential profile. The parallel wave number is also set to zero. This removes the drift-wave (requires finite parallel wave number) and the KelvinHelmholtz instability (requires sheared flow profile) branches from the solution. Pure Kelvin-Helmholtz instability is calculated using a flat density profile but a sheared $E \times B$ flow [Fig. 16(c)]. Finally, experimental profiles of density and potential [Fig. 16(d)] are used to calculate the dispersion relation for the drift-wave-Kelvin-Helmholtz (DWKH) mode. The numerical solutions of the frequencies and growth rates (both normalized to the ion cyclotron frequency $\omega_{c i}$ ) for these instabilities are shown in Figs. 16(e) and 16(f). The dispersion relation for both DWKH modes and $\mathrm{KH}$ modes agrees with the experimental data. This confirms that the Doppler-shifted frequency spectra observed in this experiment are dominated by the azimuthal flow induced by biasing. The growth rates of the DWKH modes are the largest among all cases examined. The maximum of the growth rate in DWKH mode is at $m_{\theta}=6$, which is larger but comparable to the experimental observation [ $m_{\theta} \sim 1-4$, see Figs. 12(d) and 12(g)]. The discrepancy on the mode number between the linear analysis and the experiment may be due to inaccurate modeling of dissipation, or insufficiency of using a linear model to compare with the nonlinear state measured in the experiment.

The effect of different obstacle-bias voltages on the mode growth rates for the DWKH modes are also explored using the linear model. The experimentally measured plasma potential profiles at various bias voltages in neon plasmas [Fig. 17(a)] are used in the model. Figure 17(b) shows the $\mathrm{m}$ numbers of the modes with the largest growth rate $(\gamma)$, in four cases with different bias on the annulus. The simulation result shows that the dominant mode number decreases with increasing bias. This agrees with the observation (Fig. 12) very well. 


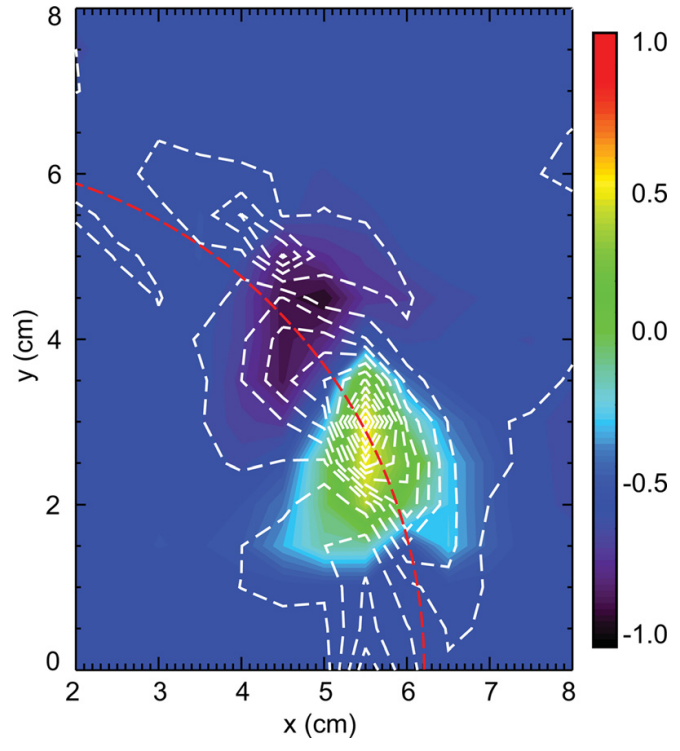

FIG. 15. (Color online) Two-dimensional cross-field correlation functions for $I_{\text {sat }}$ fluctuations (color-filled contour) and the fluctuation of the floating potential $V_{f}$ (white-dashed contour). The red dashed line indicates the edge of obstacle.

Overall, the analysis of the linear instabilities for the plasma column supports the idea that the edge turbulence is co-driven by the steep density gradient and the large sheared azimuthal flow, and that the edge $E \times B$ drift dominates the dispersion relation of the fluctuation. However, some aspects of the experiment, such as the damping rate of the high $\mathrm{m}$ number modes with large bias and the radial correlation scale of the low m modes (which leads to plasma depletion), are not correctly simulated by linear modeling. Kinetic effects might also play an important role in the instabilities,

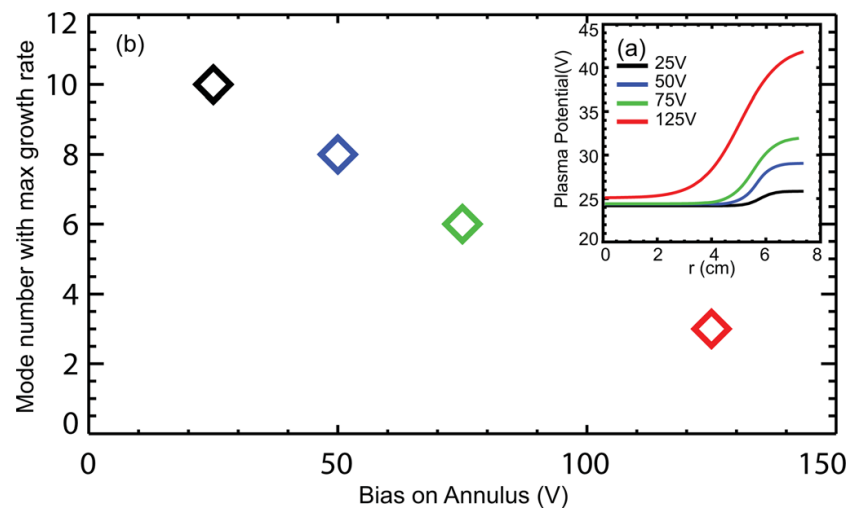

FIG. 17. (Color online). (a) Plasma potential profiles used in the linear model, measured by swept Langmuir probe. (b) Mode number ( $\mathrm{m}$ ) of modes with largest growth rate as a function of the annulus bias voltage.

especially in cases with neon plasmas and low axial magnetic fields. The boundary condition used in the linear model is periodic, thus the effect due to the fixed boundary at the LAPD end is not included. Such boundary condition may lead to a so-called "line-tying" effect, which is stabilizing the Kelvin-Helmholtz mode. ${ }^{22}$ Full nonlinear simulation (e.g., by using the BOUT (Refs. 13 and 23) three-dimensional Braginskii fluid turbulence code) is necessary to reach quantitative agreement with the experimental data.

\section{CONCLUSION}

A plasma column with $12 \mathrm{~cm}$ in diameter is formed by inserting an annular shaped obstacle concentrically with the main plasma in the LAPD. Sheared $E \times B$ flow is applied to the edge of the plasma column by biasing the obstacle. Modification of the plasma characteristics and cross-field transport

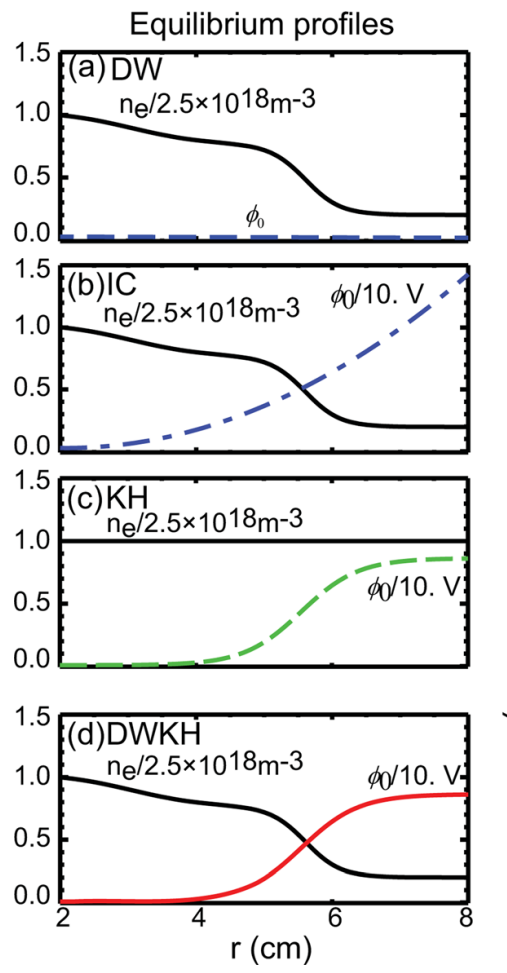

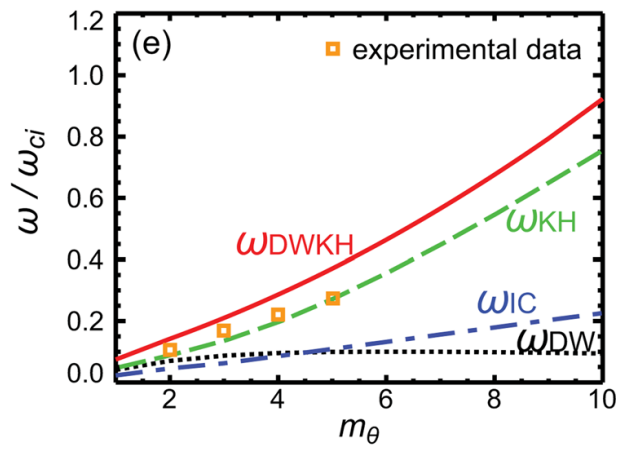

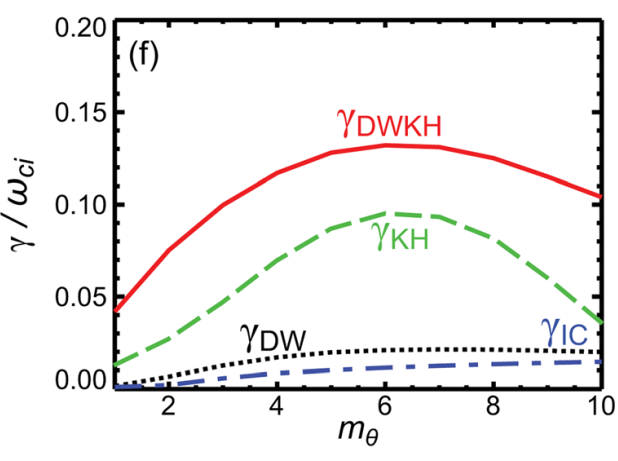

FIG. 16. (Color online) (a-c) Density and potential profiles used for calculating drift wave, interchange, and Kelvin-Helmholtz branches of the dispersion relation for the experimental parameters. (d) Experimental density and potential profiles in neon plasma with $75 \mathrm{~V}$ bias on the obstacle. (e-f) frequency and growth rate of the instabilities as a function of azimuthal mode number. The measured data are plotted in squares. 
by changing the obstacle biasing voltage, the strength of the axial magnetic field and the plasma species are fully studied.

With sheared flow induced, the most significant change in plasma characteristics is the formation of a reversed particle flux layer at the plasma edge. The reversal in transport direction is explained by the $\sim 180^{\circ}$ change in the crossphase between the density and potential fluctuation across the sheared layer, which improves plasma confinement. It is also observed that when the control parameters are above a threshold, the cross-field transport is enhanced dramatically, leading to depletion of the plasma density. In the fluctuation power spectrum, the depletion cases are associated with the dominant $m=1$ mode and the damping of the high $\mathrm{m}$ number modes with large Doppler-shift in frequency.

The experimental setup reported in this paper provides an effective way to study the modification and control of the edge plasma characteristics and edge transport, which helps to understand the basic mechanisms of the transport induced by gradient-driven and shear-driven edge turbulent waves. Several extensions of this experiment will be rich in physics and interesting. First, it is predicted that the Kelvin-Helmholtz like instabilities can be suppressed by magnetic shear. ${ }^{2}$ As a linear device, LAPD has only axial magnetic field. However, magnetic shear could be introduced experimentally, and studies on the modification of the edge turbulence by magnetic shear is practical and of great interest. Also, the diameter of the plasma column affects the edge turbulence. Studies in these aspects will help to better understand the mechanism of the sheared-flow induced confinement transition.

\section{ACKNOWLEDGMENTS}

The authors gratefully acknowledge the assistance of Shreekrishna Tripathi, Bart Van Compernolle, Marvin Drandell, Zoltan Lucky, Patrick Prybil, and Walter Gekelman for the experiment and helpful discussions. This work was supported by DOE and performed at the UCLA BaPSF basic plasma user facility supported by the NSF/DOE.

${ }^{1}$ F. Wagner, G. Becker, K. Behringer, D. Campbell, A. Eberhagen, W. Engelhardt, G. Fussmann, O. Gehre, J. Gernhardt, G. v. Gierke, G. Haas, M. Huang, F. Karger, M. Keilhacker, O. Klüber, M. Kornherr, K. Lackner, G. Lisitano, G. G. Lister, H. M. Mayer, D. Meisel, E. R. Müller,
H. Murmann, H. Niedermeyer, W. Poschenrieder, H. Rapp, H. Röhr, F. Schneider, G. Siller, E. Speth, A. Stäbler, K. H. Steuer, G. Venus, O. Vollmer, and Z. Yü, Phys. Rev. Lett. 49, 1408 (1982).

${ }^{2}$ K. H. Burrell, Phys. Plasmas 4, 1499 (1997).

${ }^{3}$ Z. Lin, T. S. Hahm, W. W. Lee, W. M. Tang, and R. B. White, Science 281, 1835 (1998).

${ }^{4}$ R. J. Taylor, M. L. Brown, B. D. Fried, H. Grote, J. R. Liberati, G. J. Morales, P. Pribyl, D. Darrow, and M. Ono, Phys. Rev. Lett. 63, 2365 (1989).

${ }^{5}$ R. R. Weynants, G. v. Oost, G. Bertschinger, J. Boedo, P. Brys, T. Delvigne, K. H. Dippel, F. Durodie, H. Euringer, K. H. Finken, D. S. Gray, J. D. Hey, D. L. Hillis, J. T. Hogan, L. Konen, R. Leners, A. M. Messiaen, A. Pospieszczyck, U. Samm, R. P. Schorn, B. Schweer, G. Telesca, R. v. Nieuwenhove, and P. E. Vandenplas, Nucl. Fusion 32, (1992).

${ }^{6}$ J. A. C. Cabral, C. A. F. Varandas, M. P. Alonso, P. Belo, R. Canário, H. Fernandes, R. Gomes, A. Malaquias, P. Malinov, F. Serra, F. Silva, and A. Soares, Plasma Phys. Controlled Fusion 40, 1001 (1998).

${ }^{7}$ G. V. Oost, J. Adámek, V. Antoni, P. Balan, J. A. Boedo, P. Devynck, I. Duran, L. Eliseev, J. P. Gunn, M. Hron, C. Ionita, S. Jachmich, G. S. Kirnev, E. Martines, A. Melnikov, R. Schrittwieser, C. Silva, J. Stöckel, M. Tendler, C. Varandas, M. V. Schoor, V. Vershkov, and R. R. Weynants, Plasma Phys. Controlled Fusion 45, 621 (2003).

${ }^{8}$ T. A. Carter and J. E. Maggs, Phys. Plasmas 16, 012304 (2009).

${ }^{9}$ J. C. Perez, W. Horton, R. D. Bengtson, and T. A. Carter, Phys. Plasmas 13, 055701 (2006).

${ }^{10}$ W. Horton, J. C. Perez, T. A. Carter, and R. Bengtson, Phys. Plasmas $\mathbf{1 2}$ 022303 (2005)

${ }^{11}$ W. Gekelman, H. Pfister, Z. Lucky, J. Bamber, D. Leneman, and J. Maggs, Rev. Sci. Instrum. 62, 2875 (1991).

${ }^{12}$ P. Popovich, M. V. Umansky, T. A. Carter, and B. Friedman, Phys. Plasmas 17, 102107 (2010).

${ }^{13}$ P. Popovich, M. V. Umansky, T. A. Carter, and B. Friedman, Phys. Plasmas 17, $122312(2010)$.

${ }^{14}$ S. Zhou, W. W. Heidbrink, H. Boehmer, R. McWilliams, T. A. Carter, S. Vincena, and S. K. P. Tripathi, Phys. Plasmas 18, 082104 (2011).

${ }^{15}$ S. L. Chen and T. Sekiguchi, J. Appl. Phys. 36, 2363 (1965).

${ }^{16}$ J. P. Gunn, C. Boucher, P. Devynck, I. Duran, K. Dyabilin, J. Horacek, M. Hron, J. Stockel, G. V. Oost, H. V. Goubergen, and F. Zacek, Phys. Plasmas 8, 1995 (2001).

${ }^{17}$ T. A. Carter, Phys. Plasmas 13, 010701 (2006).

${ }^{18}$ J. A. Boedo, P. W. Terry, D. Gray, R. S. Ivanov, R. W. Conn, S. Jachmich, G. V. Oost, and T. T. Team, Phys. Rev. Lett. 84, 2630 (2000).

${ }^{19}$ C. Holland, J. H. Yu, A. James, D. Nishijima, M. Shimada, N. Taheri, and G. R. Tynan, Phys. Rev. Lett. 96, 195002 (2006).

${ }^{20}$ F. Brochard, E. Gravier, and G. Bonhomme, Phys. Plasmas 12, 062104 (2005).

${ }^{21}$ S. I. Braginskii, in Reviews of Plasma Physics, edited by M. A. Leontovich (Consultants Bureau, New York, 1965), pp. 205-311.

${ }^{22}$ A. Miura and J. R. Kan, Geophys. Res. Lett. 19, 1611, doi:10.1029/ 92GL01448 (1992).

${ }^{23}$ M. V. Umansky, X. Q. Xu, B. Dudson, L. L. LoDestro, and J. R. Myra, Commun. Comput. Phys. 180, 887 (2009). 